\title{
World Modeling, Integrated Information, and the Physical Substrates of Consciousness; Hidden Sources of the Stream of Experience?
}

\author{
Adam Safron ${ }^{1,2,3}$ \\ ${ }^{1}$ Center for Psychedelic and Consciousness Research, Johns Hopkins University School of \\ Medicine, Baltimore, MD 21218, USA \\ ${ }^{2}$ Kinsey Institute, Indiana University, Bloomington, IN 47405, USA \\ ${ }^{3}$ Cognitive Science Program, Indiana University, Bloomington, IN 47405, USA \\ asafron@gmail.com
}

\begin{abstract}
In this brief commentary on The Hidden Spring: A Journey to the Source of Consciousness, I describe ways in which Mark Solms' account of the origins of subjective experience relates to Integrated World Modeling Theory (IWMT). IWMT is a synthetic theory that brings together different perspectives, with the ultimate goal of solving the enduring problems of consciousness, including the Hard problem. I describe points of compatibility and incompatibility between Solms' proposal and IWMT, with particular emphasis on how a Bayesian interpretation of Integrated Information Theory and Global (Neuronal) Workspace Theory may help identify the physical and computational substrates of consciousness.
\end{abstract}

\section{Contents}

Of MICS/MICE and generative models ....................................................................................

Hard problems regarding the physical substrates of consciousness .............................................5

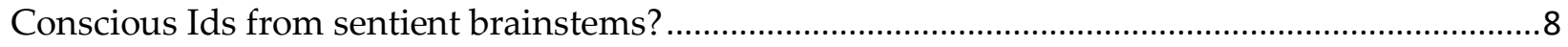

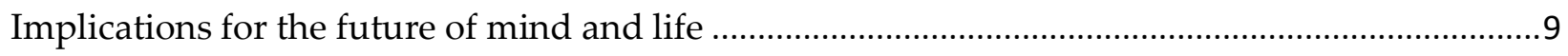

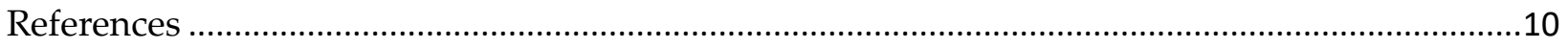

In The Hidden Spring: A Journey to the Source of Consciousness (THS), Mark Solms (2021) compellingly describes how recognizing the fundamental continuity between mind and life may allow us to understand the nature(s) of conscious phenomena. THS also describes a personal journey driven by a desire to obtain a scientific understanding of the nature(s) of human meanings. Solms' deeply engrossing book further draws upon insights from his pioneering work founding the discipline of neuropsychoanalysis, wherein minds are studied with both the richness of psychodynamic models and empirical grounding of neuroscience. By resisting longstanding taboos for seriously contemplating consciousness and psychoanalytic ideas, THS helps reconcile the "manifest image" of personal experience and the "scientific image" of abstract theory (Dennett, 2017). By creating richly detailed connections between 
subjective and objective perspectives on consciousness, THS makes substantial progress in bridging explanatory gaps between mind and body-brain.

Both in terms of existential motivations and inspirations from the Free Energy Principle and Active Inference (FEP-AI) framework (Friston et al., 2017), much of Solms' account resonates with my own attempts at understanding consciousness (Safron, 2020a, 2020b, 2020c, 2021a, 2021b). With Integrated World Modeling Theory (IWMT), I draw upon FEP-AI to create a synthetic perspective that combines aspects of Integrated Information Theory (IIT) (Tononi et al., 2016) and Global (Neuronal) Workspace Theory (G(N)WT) (Baars et al., 2013; Dehaene, 2014). ["G(N)WT" is used to indicate a hybrid perspective drawing upon both Baars' original proposal and its later extensions by Dehaene.] At its core, IWMT suggests phenomenal consciousness (P-C) is what it is like to be the functioning of a generative model over the sensorium of an embodied, environmentally-embedded agent, iteratively estimating (or filling-in) data from likely systemworld states, given past experience. This is highly compatible with THS, except Solms emphasizes the generation of feelings from phylogenetically ancient midbrain structures as the primary and sufficient source of P-C, so shaping all other forms of modeling in the service of evolutionary fitness (i.e., survival and reproduction as biological value). Below I describe how IWMT agrees with the fundamental importance of affects for constraining the evolution of consciousness, while also suggesting that such affective phenomena likely require cortical rerepresentation to serve the functions described in THS.

IWMT suggests that generative modeling processes may only bring forth worlds of experience if they afford sufficient degrees of coherence for space (degrees of locality), time (relative changes in space), and cause (regularities in these changes), all of which likely require minimal agentic selfhood. For any kind of world to appear to a modeling system, it must be able to situate things relative to other things with specific spatiotemporal and causal properties; otherwise, there would be no experienceable world. Put differently, Kantian synthetic a priori categories may be more than preconditions for coherent judgment (De Kock, 2016; Northoff, 2012; Swanson, 2016); rather, these coherence-making "intuitions" may be required for any kind of sense-making whatsoever with respect to the conscious access (C-A) of experience (Block, 2008), and possibly for P-C as well. [While "access consciousness" is a more common use of terminology, here I use the expression C-A in agreement with IIT's position that such forms of knowledge are only experienced via P-C.] This could be compatible with THS, except below I describe how brainstem processes may be insufficient for realizing this coherent structuring of experience, which could preclude their sufficiency as sources of P-C.

To date, no theory is generally accepted as constituting a sufficient explanation for consciousness. However, IWMT claims a "minimum unifying model" (MUM) (Wiese, 2020) may be obtained by combining FEP-AI's probabilistic modeling and cybernetic grounding, IIT's characterization of intrinsically-defined systems, and G(N)WT's workspaces for informational synergy. In this commentary, I mostly focus on IIT, which plays dual roles in IWMT: a) as a modeling framework for emergent phenomena with complementarity with FEP-AI; b) as part of a unified theory of consciousness with complementarity with G(N)WT. THS could provide yet another essential perspective for this synthesis: consciousness as we know it is fundamentally grounded in life management, with all cognition being understood as extended allostasis (i.e., predictive/proactive maintenance of homeostatic boundaries). However, below I describe 
potential obstacles for integrating Solms' thinking into IWMT, focusing on the following questions:

1. Is consciousness capable of being sufficiently realized by brainstem processes?

2. Do affects entail consciousness in their own right, or do they involve forms of cortical rerepresentation?

\section{Of MICS/MICE and generative models}

IIT begins by identifying axioms regarding features shared across all experiences (Tononi et al., 2016), which are then translated into postulated mechanisms capable of realizing these experiential properties. [While IIT argues these physical mechanisms are sufficient for realizing consciousness, IWMT and THS would suggest these properties may only be necessary conditions, thereby limiting more quasi-panpsychist implications.] Degrees of integrated information (phi, or self-cause-effect power) can be (theoretically) computed for any candidate system, but only integrated information maxima (Phi) qualify as intrinsically existing conscious systems. A maximally irreducible cause-effect structure (MICS) is hypothesized to correspond to a physical substrate of consciousness (PSC), constituting the totality of experience evolving over a particular temporal and spatial grain, within which particular qualitative distinctions can be identified as maximally irreducible cause-effect repertoires (MICE). This process for identifying potentially conscious systems may be compatible with both THS and IWMT, except MICS/MICE would entail consciousness if-and-only-if given semantic grounding via coherent world modeling. Consciousness may be entailed by these mechanisms because neural dynamics are coupled to sensors/effectors, which are further coupled to coherent system-world dynamics, so affording modeling of those coherences.

With respect to emergence, IWMT identifies correspondences between IIT's procedure for identifying intrinsically-existing systems (as MICS/MICE) and FEP-AI's definition of systems in terms of Markov blanket (MB) boundaries (Friston et al., 2020). Further parallels are identified between MICS/MICE as nexuses of self-cause-effect-power and MB-bound systems as generating model evidence for their existence via prediction-error-minimization. Drawing upon the Harmonic Brain Modes framework (Atasoy et al., 2018), IWMT describes MB-bound MICS/MICE as "self-organizing harmonic modes" (SOHMs), which promote "communication through coherence" (Deco \& Kringelbach, 2016), thereby implicitly computing joint marginal probability distributions over networks within their scope. If such joint beliefs come from world models with sufficient spatiotemporal and causal coherence, then they may entail consciousness according to IWMT.

IWMT further identifies correspondences between IIT's MICS/MICE (as MB-bound SOHMs) and workspaces/modules in G(N)WT. With relevance for identifying candidate PSCs, variational autoencoding provides an algorithmic description of cortical functioning, wherein generative models are trained to infer likely observations by learning to reconstruct data passed (or compressed) through dimensionality-reducing bottlenecks (Schmidhuber, 2020, 2021). While each sensory modality constitutes its own autoencoding hierarchy, efficient synergistic inference/learning is enabled when reduced-dimensionality subnetworks are connected into a shared latent (work) space (McEliece et al., 1998). From this perspective, particular conscious contents are generated based on the extent of SOHM-mediated recurrent computation 
within/between various sensory hierarchies (Figure 1). The hierarchically-organized brainstem processes emphasized by THS could be understood as forms of (predictive) autoencoding via generalized synchrony with informational bottlenecks (Lahav et al., 2018; Lu \& Bassett, 2020; Tishby \& Zaslavsky, 2015); however, cortical computation may be uniquely well-suited to implement world modeling with sufficient coherence to realize consciousness.

On account of these computational principles and relative locations for sensory hierarchies, IWMT suggests alpha-synchronized SOHMs over posterior association cortices support largescale workspaces for perceptual (and imaginative) inference. Coherent egocentric perspective may be established as posterior alpha rhythms integrate head-direction/body-pose information from respective posterior-midline/lateral-parietal cortices. These subsystems are suggested to provide respective sources of visuospatial and somatospatial awareness, which when coupled may entail spatiotemporally-coherent world models with a "lived body" at their center (Rudrauf et al., 2017). This is consistent with IIT's claim that a "posterior hot zone" (PHZ) constitutes the PSC (Boly et al., 2017), as well as G(N)WT's claim that frontal lobes are required for various forms of C-A (conscious access) via larger workspaces with more sophisticated modeling capacities (Safron, 2021a). Thus, IWMT (and perhaps THS) may help resolve the longstanding neuroanatomical debate between IIT and G(N)WT by allowing both theories to be accurate, but regarding different modes of conscious experiences (i.e., P-C vs. C-A).
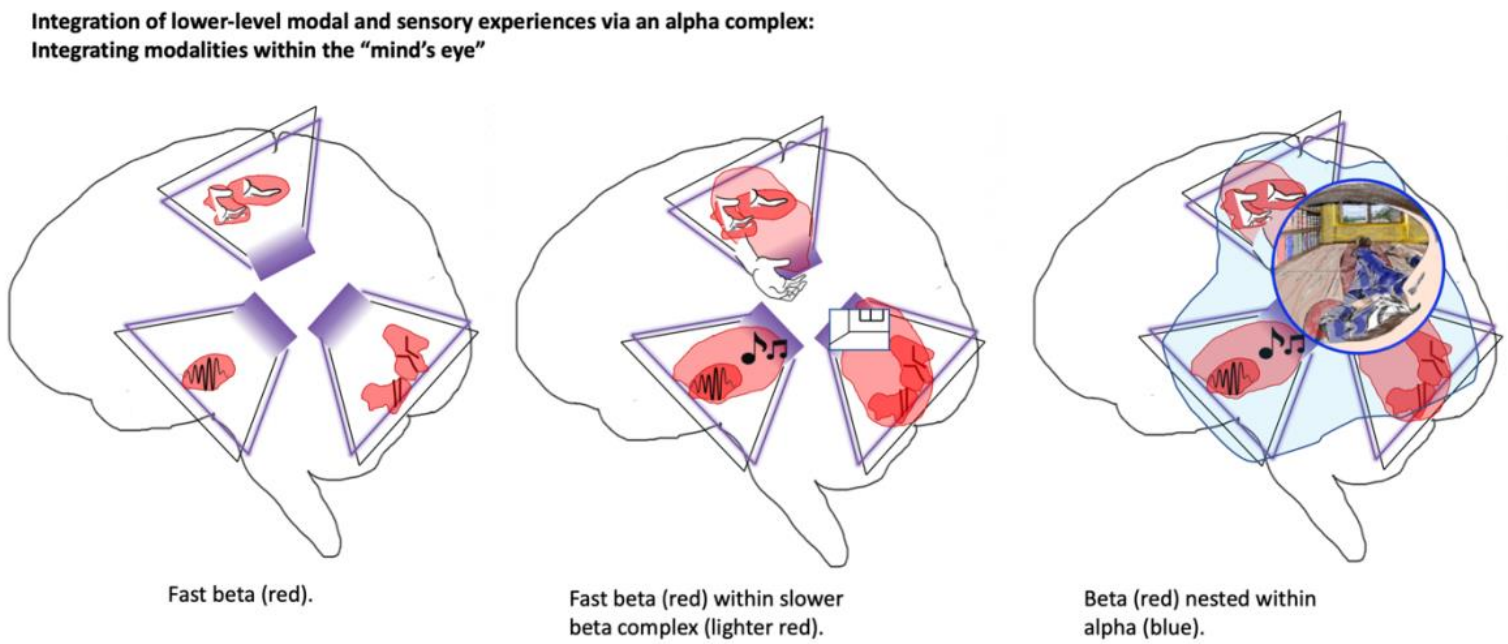

Figure 1: Integration of sense data into a coherent field of experience. Predictive processing cortical hierarchies are depicted as trapezoidal shapes (Safron, 2020a, 2020c, 2021b). Smaller-and-faster or largerand-slower forming beliefs are communicated at respective lower $(\sim 13-20 \mathrm{~Hz})$ or higher $(\sim 20-30 \mathrm{~Hz})$ beta frequencies. Smaller and faster rhythmic complexes are depicted as nested within larger and more slowly unfolding rhythms, so enabling hierarchical modeling of complex events over multiple spatiotemporal scales. Larger and more slowly unfolding alpha rhythms $(\sim 7-12 \mathrm{~Hz})$ provide yet another level of hierarchical depth for deep temporal modeling, organized according to coherent egocentric reference 
frames. From left to right, panels depict a progression moving from modeling lower-level features to more complex compositions, and finally integration into a coherent conscious field. This visuospatial sketchpad-and "mind's eye," or "Cartesian theater" - is likely heavily dependent upon posterior midline structures that constitute major loci for alpha synchronization and sources of visual imagery/ awareness (Xie et al., 2020). While not depicted, somatic consciousness may be generated primarily via lateral parietal cortices, which may couple with visuospatial modalities in complex ways.

\section{Hard problems regarding the physical substrates of consciousness}

THS and IWMT both reject more panpsychist views, such as IIT's suggestion that consciousness is "what physics feels like from the inside" (Koch, 2012). This statement is partially consistent with FEP-AI's claim that generative modeling principles linking brains and minds also link all persisting systems with their dynamics. Thus, FEP-AI is panprotopsychist (Friston et al., 2020) in arguing that systems must exhibit certain mind-like qualities to temporarily resist the $2^{\text {nd }}$ law. However, both THS and IWMT restrict consciousness to lifeforms with complex nervous systems. A relevant FEP-AI distinction may be found in the distinction between "being a model" and "having a model" (Ramstead et al., 2019; Seth \& Tsakiris, 2018):

- Being models: characterization of interactions between organisms and their niches as generative processes.

- Having models: having subsystems capable of modeling these generative processes. According to IWMT, experience only emerges from systems having models capable of generating spatiotemporally and causally coherent inferences over system-world states.

THS and IWMT agree regarding understanding nervous systems in terms of their primary evolutionary functions for adaptively shaping behavior. Models/representations may be identified when action-perception cycles have meaningfully informative correspondences with attracting states of the (proactively) reactive nexus. However, for there to be "something that it feels like," modeling processes must generate representations of coherent organismcentered lifeworlds (Shanahan, 2010; Tani, 2016), with time-evolving state transitions representing inferential filling-in of likely sensory states. IWMT further suggests this modeling requires workspaces as dynamic cores of effective connectivity with high degrees of integrated information, so affording inferences with sufficient rapidity for informing (and being informed by) action-perception cycles whereby systems adaptively couple with the world. In addition to requiring access to richly connected subnetworks (Heuvel \& Sporns, 2011), such modelselection may require additional (potentially cortex-requiring) properties:

1. Fluctuating periods of local and global modularity for iterative simulated annealing via dual-phase evolution (Kiefer, 2020; Paperin et al., 2011; Shine, 2019; Strindberg et al., 2021).

2. Sufficiently large networks for inner-loop processes to exhibit (temporarily) separate evolution from immediate environmental couplings, so affording the self-reflexive modeling and counterfactual processing necessary for adaptive active inference (Corcoran et al., 2020; Kirchhoff et al., 2018).

If(-and-only-if) such properties are realizable within a particular system, dynamics could potentially entail sufficiently coherent integrated self-world modeling for generating likely patterns of sense data (i.e., the stream of experience). 
How can there be "something that it is/feels like" to be a physical system? The language of this Hard question may be suggestive of potential answers, with "something" suggesting modelable 'things', "is/feels" suggesting the centrality of embodied experience, and "like" suggesting the informative nature of conscious states (as Bayesian empirical prior expectations) with degrees of similarity/difference with other possible experiences (Safron, 2019). This is all consistent with THS and IWMT, where both theories attempt to provide a multi-level account of experience spanning functional, algorithmic, and implementational levels of analysis (Marr, 1983). Further, both THS and IWMT argue that other theories such as IIT and G(N)WT require semantic grounding via cybernetic meaning-making, with brains understood as control systems for embodied-embedded agents.

Yet THS and IWMT substantially differ with respect to algorithmic and implementational-level details regarding PSCs (physical substrates of consciousness) (Figure 2). While THS focuses on brainstems, IWMT (largely in agreement with IIT) focuses on rhythmic complexes centered on posterior cortices as realizers of subjective experience. THS describes consciousness as a process for influencing belief dynamics by subjectively-felt drives based on the importance of information, with such attentional selection understood as "precision weighting" in FEP-AI (Friston et al., 2017). Attention and consciousness are intimately related in IWMT and other theories (Graziano, 2019), and conscious representation of affective states is crucial for adaptively shaping belief dynamics to realize biological value (Safron, 2021a). Further, well-calibrated tuning of Bayesian updating is certainly necessary for achieving the forms of coherence emphasized by IWMT and G(N)WT more generally (Shea \& Frith, 2019). Even more, re-entrant signaling patters in "dynamic core" workspace models may themselves be understood as self-attention (Baars et al., 2013; Bach, 2019; Goyal et al., 2021; VanRullen \& Kanai, 2021), consistent with THS' view of consciousness as process for precision-weighting based on organismic value.

Yet, in contrast to THS' conscious brainstems/"Id", IWMT suggests affective consciousness may require cortical re-representation. More specifically, affects may require cross-modal mappings to achieve sufficient spatiotemporal coherence for conscious modeling. Theoretically, restricted localizability of interoceptive sensations may contribute to quasisynesthetic percepts in which they are 'infused' into other modalities (Safron, 2021a), potentially contributing to various forms of phenomenal binding. The life-centered view of THS is highly compatible with this widely distributed and functionally central account of interoceptive inference, including with respect to conscious awareness (Huang et al., 2021). However, IWMT differs from THS in suggesting that not only may brainstems be unconscious on their own, but even insula-mediated interoception may only attain to consciousness via influencing belief dynamics centered on posterior-medial/lateral-parietal cortices. Nonetheless, IWMT and THS concur that affectively-shaped precision-weighting of action/perception cycles may be a primary evolutionary function of consciousness.

IWMT originally suggested that conscious contents may be determined by the extent of alpha-synchronized complexes over different levels of distributed feature hierarchies in posterior cortices (Safron, 2020a). However, alpha rhythms could instead indicate passive sampling of the environment and organization by egocentric reference frames, but with consciousness being updated more quickly via beta-synchronized subnetworks. IWMT further 
proposes that cortical workspaces may operate according to principles of geometric deep learning (Safron, 2020b, 2020b, 2021a, 2021b; Zhou et al., 2019), with chronotopic and potentially semi-topographic correspondences between neural-activity-manifolds and the details of subjective experience. This proposal was primarily suggested because of the extreme efficiency of graph neural networks for modeling physical processes with homomorphic geometries. If accurate, PSCs could have an even more circumscribed extent, potentially restricted to fast beta (and possibly gamma) signaling within cortical hubs such as posterior-midline/lateral-parietal cortices. Alternatively, rapid inferences from those subnetworks could themselves be unconscious, yet afford coherent orchestration of conscious inferential dynamics distributed throughout sensory hierarchies (e.g. intelligent sampling from autoencoding latent spaces).

Until such possibilities can be further constrained, hard questions remain regarding the Hard problem. With respect to THS, the superior colliculus may possess homomorphic geometries over attentional states, and might even induce quasi-topographic geometric representations in posterior midline structures. However, without supporting spatiotemporallycoherent modeling of objects, IWMT suggests the superior colliculus would be insufficient for generating consciousness.

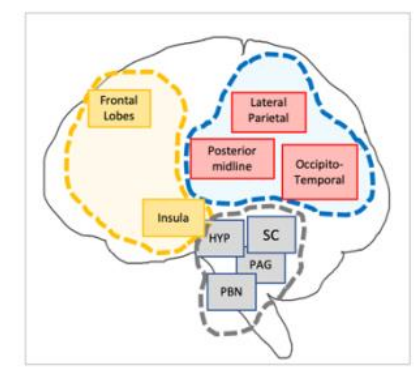

1. Many Minds

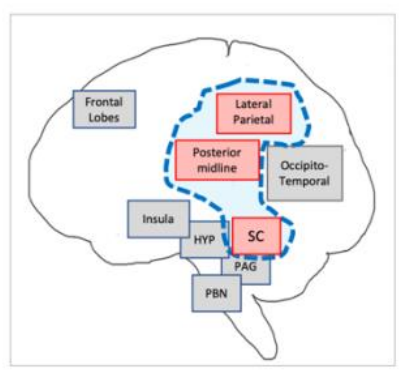

3. Geometric IWMT

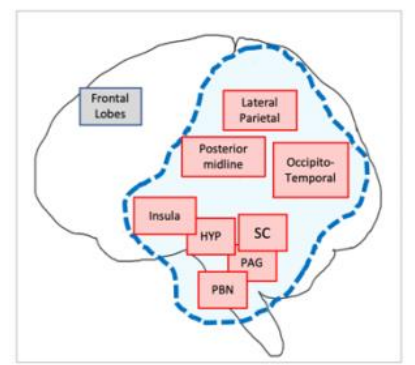

2. Allostatic IWMT

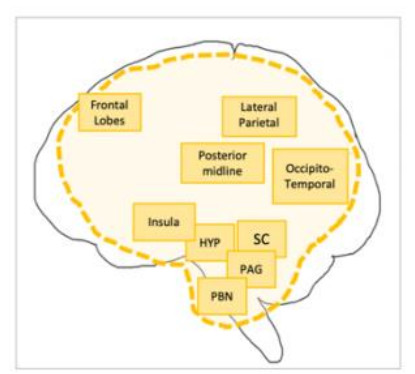

4. Allostatic G(N)WT
Abbreviations

HYP: Hypothalamus

SC: Superior colliculus

PAN: Paraquedual grey

Figure 2: Possibilities for realizing phenomenal consciousness (P-C) and conscious access (C-A) as suggested by THS and other theories. 1) "Many Minds" depicts the posterior hot zone (PHZ), brainstem, and perhaps frontal lobes (and insula) as all entailing separate P-C sources; this would be compatible with both THS and IIT, but with only the PHZ associated with conscious processing in IWMT. 2) "Allostatic IWMT" depicts P-C via a complex spanning brainstem and posterior cortices, which could also be THS-compatible. However, IIT suggests integrated information for highly (meaningfully) self-entangled PHZs would decrease if including other systems; IWMT is agnostic on this issue, as substantial functional coupling could be established via rapidly changing neuromodulation and mechanisms such as zero-lag phase synchronization (Gollo et al., 2014; Shine et al., 2019). 3) "Geometric IWMT" depicts another possibility with high degrees of coupling between (semi-)topographic representations of PHZ-subsets and the superior colliculus, but with other systems not contributing to generating consciousness within spatiotemporal scales over which such computations are realized; this could be compatible with IIT, but possibly not THS. 4) "Allostatic $\mathrm{G}(\mathrm{N}) W T^{\prime \prime}$ depicts an IWMT-compatible scenario in which interoceptive information from brainstem influences C-A, 
and thereby indirectly P-C; this would be compatible with other theories of consciousness, including IIT iff P-C is defined as experience itself, and with C-A reflecting P-C contributing to various forms of higher-order cognition.

\section{Conscious Ids from sentient brainstems?}

THS argues for subcortically-mediated feeling awareness as the primary form of consciousness, drawing on evidence from brainstem stimulation as sufficient generator of affective states, emotional expressions in anencephalic patients, and preserved feelings with bilateral insula damage. With respect to this putative evidence for conscious brainstems, the following counterarguments may be suggested:

1. Cortical processes could mediate conscious-affect-generation from brainstem stimulation (Barrett, 2017; LeDoux, 2019).

2. We cannot know what anencephalic patients experience. If emotions/feelings are understood as largescale organismic modes for coherent adaptive behavior - potentially understood as abstract action/perception cycles (Safron, 2021a) - then affective displays could be realized without consciousness. It should be further remembered that intelligent behavior can sometimes be achieved through simple means (Lind, 2018; Taylor et al., 2012; Vasas \& Chittka, 2019) - including cleverly designed body plans (Pfeifer \& Bongard, 2006) - rather than via (potentially-conscious) sophisticated models.

3. Interoceptive inference may primarily centre on the insular hierarchy early in development, yet become more broadly distributed through predictive coding mechanisms. Further, much feeling may not be purely interoceptive, but may also involve proprioceptive and exteroceptive dynamics, such as body-posture modifications - perhaps especially for the face and hands - with particular organismic and ecological significances (Sandrone \& Safron, 2013). Hence, cortical-based emotions need not imply affective consciousness removal with insula damage (Damasio et al., 2013).

Some perhaps uncomfortable analogies could be drawn between anencephalic patients and our more phylogenetically basal cousins such as reptiles and fish. These phyla have cortical homologues capable of supporting body maps (Ocaña et al., 2015; Suryanarayana et al., 2017), yet is unclear whether there are reptile or fish species whose palliums have sufficient hierarchical complexity for coherent world modeling. Theoretically, P-C could be sufficiently enabled by coherently-organized body-map state-transitions, orchestrated according to spatiotemporal trajectories of hippocampal/entorhinal systems (Çatal et al., 2021). It is not inconceivable that such sequentially-inferred somatic states could be accompanied by P-C, even without contextualization within integrated world models possessing "temporal depth" and "counterfactual richness" (Friston, 2018). Such embodied inference would be insufficient for establishing causal reasoning and flexible modeling, but could nonetheless have sufficient coherence for generating P-C. This could push the advent of consciousness as far back as 560 million years ago with our common ancestors with lampreys (Suryanarayana et al., 2020), and possibly earlier.

From the FEP-AI perspective described above-with respect to being/having models brainstem attracting states could entail generative modeling of extended phenotypes and affectively-driven niche-construction processes (Ramstead et al., 2019; Seth \& Tsakiris, 2018). However, it is unclear whether such generative processes are separable from the implicit 
modeling realized by the overall action of the organism and its environmental couplings, wherein organism-environment systems are models in their very being/enacting. That is, if we grant consciousness to brainstem structures, we may also have to admit something-that-it-islike-ness for extra-neural and even extra-physiological processes (Clark \& Chalmers, 1998).

At higher levels of hierarchical organization, brainstems could conceivably have internal attractors whose dynamics come to represent organism-environment interactions, so providing further levels of predictive control. The superior colliculus does support (egocentric) spatial "salience maps" via an organismic hierarchy over the parabrachial nucleus, nucleus tractus solitarus, and periaqueductal gray (Damasio, 2012). Yet, enslavement by immediate sensorimotor contingencies might preclude the kinds of dual-phase evolution with counterfactual/reflective modeling suggested above as potentially necessary conditions for coherently estimating system-world states. This would preclude brainstem processes from modeling the overall organismic system with sufficient spatiotemporal/causal coherence for consciousness, let alone providing sufficient bases for active inference based on evaluating the expected consequences of selecting different actions in the (counterfactual) future.

According to IIT, the PHZ (posterior hot zone) likely constitutes a MICS/MICE (maximally irreducible cause-effect structure/repertoires) due to its connectomic properties as a hierarchy of stacked grids, which produce higher degrees of integrated information (phi) relative to less organized recurrent systems. Brainstems would therefore be excluded from a MICS/MICE, since its inclusion would generate less Phi. However, IIT analyses would likely identify brainstems (or set of substructures) as separate conscious systems on their own (Figure 2), potentially with THS' affective conceptual distinctions as various MICE repertoires. While likely incompatible with IWMT, IIT may further identify frontal cortices as separable conscious systems in addition to PHZs and brainstems, the functions of which - with perhaps extreme poetic license-could respectively be likened to aspects of superegos, egos, and Ids in Freud's account of psyches governed by interactions between otherwise separate causal powers (Carhart-Harris \& Friston, 2010). IWMT could support this psychodynamic perspective, except such psychodynamic quasi-agents would likely be realized as separable (self-organizing harmonic) modes over distributed neurodynamic processes, while not necessarily involving clear modularity.

\section{Implications for the future of mind and life}

Analyses of infant brains suggest that integrated information (Phi) increases over the course of development, with major transitions for increased Phi occurring at approximately 3540 weeks of age (Isler et al., 2018). While the intelligence of infants may be grossly underestimated (Gopnik, 2009), it is unclear when infants actually acquire capacities for coherent world modeling. Some indirect evidence for infant consciousness is provided by observations of P300-like waveforms and global ignition as emphasized by G(N)WT (Dehaene, 2014). Yet, if IWMT is accurate regarding potentially complex coherence-making properties being necessary for realizing consciousness, we may find ourselves in ethically complicated positions where infants are unconscious until some indeterminate time after birth. Further, if THS is accurate regarding conscious brainstems, we may also find ourselves in ethically complicated positions of identifying consciousness in unborn fetuses. However, both THS and 
IWMT would sidestep another set of ethical complications in suggesting cerebral organoids to be unconscious (Bayne et al., 2019), unless given complex optimization as cybernetic control systems for embodied-embedded agents.

THS ends with an intriguing (and potentially troubling) exploration of how artificial consciousness could be realized by recapitulating functional principles of brainstems as allostatic controllers (Man \& Damasio, 2019; Solms, 2021). IWMT, however, suggests that artificial thalamocortical systems are likely required for realizing such conscious machines, and IIT would further argue that even full brain emulations would be unconscious if deployed on computing architectures involving serial physical operations (Tononi \& Koch, 2015). IWMT is thus more inclusive than IIT and less inclusive than THS with respect to sufficient realizers of consciousness, as integrated information (as cause-effect power) could potentially be maximal for temporally extended processes including virtual machines (Safron, 2020a, 2021b), which could afford consciousness-entailing forms of informational closure (Chang et al., 2019), even without (albeit likely practically necessary) neuromorphic computing platforms. If we do attempt to create conscious AI (Bengio, 2017; Koch, 2019; Safron, 2020b, 2021b; Tegmark, 2017) - and potentially succeed in this endeavor - then such intellectual debates may ultimately have an importance that is difficult to imagine.

\section{References}

Atasoy, S., Deco, G., Kringelbach, M. L., \& Pearson, J. (2018). Harmonic Brain Modes: A Unifying Framework for Linking Space and Time in Brain Dynamics. The Neuroscientist: A Review Journal Bringing Neurobiology, Neurology and Psychiatry, 24(3), 277293. https://doi.org/10.1177/1073858417728032

Baars, B. J., Franklin, S., \& Ramsoy, T. Z. (2013). Global Workspace Dynamics: Cortical “Binding and Propagation” Enables Conscious Contents. Frontiers in Psychology, 4. https://doi.org/10.3389/fpsyg.2013.00200

Bach, J. (2019). The Cortical Conductor Theory: Towards Addressing Consciousness in AI Models. In A. V. Samsonovich (Ed.), Biologically Inspired Cognitive Architectures 2018 (pp. 16-26). Springer International Publishing. https://doi.org/10.1007/9783-319-99316-4_3

Barrett, L. F. (2017). How Emotions Are Made: The Secret Life of the Brain. Houghton Mifflin Harcourt.

Bayne, T., Seth, A. K., \& Massimini, M. (2019). Are There Islands of Awareness? Trends in Neurosciences, 0(0). https://doi.org/10.1016/j.tins.2019.11.003

Bengio, Y. (2017). The Consciousness Prior. ArXiv:1709.08568 [Cs, Stat]. http://arxiv.org/abs/1709.08568

Block, N. (2008). Phenomenal and Access Consciousness Ned Block and Cynthia MacDonald: Consciousness and Cognitive Access. Proceedings of the Aristotelian Society, 108, 289-317. JSTOR.

Boly, M., Massimini, M., Tsuchiya, N., Postle, B. R., Koch, C., \& Tononi, G. (2017). Are the Neural Correlates of Consciousness in the Front or in the Back of the Cerebral Cortex? Clinical and Neuroimaging Evidence. Journal of Neuroscience, 37(40), 96039613. https://doi.org/10.1523/JNEUROSCI.3218-16.2017

Carhart-Harris, R. L., \& Friston, K. J. (2010). The default-mode, ego-functions and free-energy: A neurobiological account of Freudian ideas. Brain: A Journal of Neurology, 133(Pt 4), 1265-1283. https://doi.org/10.1093/brain/awq010

Çatal, O., Verbelen, T., Van de Maele, T., Dhoedt, B., \& Safron, A. (2021). Robot navigation as hierarchical active inference. Neural Networks, 142, 192-204. https://doi.org/10.1016/j.neunet.2021.05.010

Chang, A. Y. C., Biehl, M., Yu, Y., \& Kanai, R. (2019). Information Closure Theory of Consciousness. ArXiv:1909.13045 [q-Bio]. http://arxiv.org/abs/1909.13045

Clark, A., \& Chalmers, D. J. (1998). The Extended Mind. Analysis, 58(1), 7-19.

Corcoran, A. W., Pezzulo, G., \& Hohwy, J. (2020). From allostatic agents to counterfactual cognisers: Active inference, biological regulation, and the origins of cognition. Biology \& Philosophy, 35(3), 32. https://doi.org/10.1007/s10539-020-09746-2

Damasio, A. (2012). Self Comes to Mind: Constructing the Conscious Brain (Reprint edition). Vintage.

Damasio, A., Damasio, H., \& Tranel, D. (2013). Persistence of Feelings and Sentience after Bilateral Damage of the Insula. Cerebral Cortex (New York, NY), 23(4), 833-846. https://doi.org/10.1093/cercor/bhs077

De Kock, L. (2016). Helmholtz's Kant revisited (Once more). The all-pervasive nature of Helmholtz's struggle with Kant's Anschauung. Studies in History and Philosophy of Science, 56, 20-32. https://doi.org/10.1016/j.shpsa.2015.10.009 
Deco, G., \& Kringelbach, M. L. (2016). Metastability and Coherence: Extending the Communication through Coherence Hypothesis Using A Whole-Brain Computational Perspective. Trends in Neurosciences, 39(3), 125-135. https://doi.org/10.1016/j.tins.2016.01.001

Dehaene, S. (2014). Consciousness and the Brain: Deciphering How the Brain Codes Our Thoughts. Viking.

Dennett, D. (2017). From Bacteria to Bach and Back: The Evolution of Minds (1 edition). W. W. Norton \& Company.

Friston, K. J. (2018). Am I Self-Conscious? (Or Does Self-Organization Entail Self-Consciousness?). Frontiers in Psychology, 9. https://doi.org/10.3389/fpsyg.2018.00579

Friston, K. J., FitzGerald, T., Rigoli, F., Schwartenbeck, P., \& Pezzulo, G. (2017). Active Inference: A Process Theory. Neural Computation, 29(1), 1-49. https://doi.org/10.1162/NECO_a_00912

Friston, K. J., Wiese, W., \& Hobson, J. A. (2020). Sentience and the Origins of Consciousness: From Cartesian Duality to Markovian Monism. Entropy, 22(5), 516. https://doi.org/10.3390/e22050516

Gollo, L. L., Mirasso, C., Sporns, O., \& Breakspear, M. (2014). Mechanisms of zero-lag synchronization in cortical motifs. PLoS Computational Biology, 10(4), e1003548. https://doi.org/10.1371/journal.pcbi.1003548

Gopnik, A. (2009). The Philosophical Baby: What Children's Minds Tell Us About Truth, Love, and the Meaning of Life. Macmillan.

Goyal, A., Didolkar, A., Lamb, A., Badola, K., Ke, N. R., Rahaman, N., Binas, J., Blundell, C., Mozer, M., \& Bengio, Y. (2021). Coordination Among Neural Modules Through a Shared Global Workspace. ArXiv:2103.01197 [Cs, Stat]. http://arxiv.org/abs/2103.01197

Graziano, M. S. A. (2019). Rethinking consciousness: A scientific theory of subjective experience (First Edition.). WWNorton \& Company.

Heuvel, M. P. van den, \& Sporns, O. (2011). Rich-Club Organization of the Human Connectome. The Journal of Neuroscience, 31(44), 15775-15786. https://doi.org/10.1523/JNEUROSCI.3539-11.2011

Huang, Z., Tarnal, V., Vlisides, P. E., Janke, E. L., McKinney, A. M., Picton, P., Mashour, G. A., \& Hudetz, A. G. (2021). Anterior insula regulates brain network transitions that gate conscious access. Cell Reports, 35(5). https://doi.org/10.1016/j.celrep.2021.109081

Isler, J. R., Stark, R. I., Grieve, P. G., Welch, M. G., \& Myers, M. M. (2018). Integrated information in the EEG of preterm infants increases with family nurture intervention, age, and conscious state. PloS One, 13(10), e0206237. https://doi.org/10.1371/journal.pone.0206237

Kiefer, A. B. (2020). Psychophysical identity and free energy. Journal of The Royal Society Interface, 17(169), 20200370. https://doi.org/10.1098/rsif.2020.0370

Kirchhoff, M., Parr, T., Palacios, E., Friston, K. J., \& Kiverstein, J. (2018). The Markov blankets of life: Autonomy, active inference and the free energy principle. Journal of the Royal Society Interface, 15(138). https://doi.org/10.1098/rsif.2017.0792

Koch, C. (2012). Consciousness: Confessions of a Romantic Reductionist. MIT Press.

Koch, C. (2019). The Feeling of Life Itself: Why Consciousness Is Widespread but Can't Be Computed. MIT Press.

Lahav, N., Sendiña-Nadal, I., Hens, C., Ksherim, B., Barzel, B., Cohen, R., \& Boccaletti, S. (2018). Synchronization of chaotic systems: A microscopic description. Physical Review E, 98(5), 052204. https://doi.org/10.1103/PhysRevE.98.052204

LeDoux, J. (2019). The Deep History of Ourselves: The Four-Billion-Year Story of How We Got Conscious Brains. Viking.

Lind, J. (2018). What can associative learning do for planning? Royal Society Open Science. https://doi.org/10.1098/rsos.180778

Lu, Z., \& Bassett, D. S. (2020). Invertible generalized synchronization: A putative mechanism for implicit learning in neural systems. Chaos (Woodbury, N.Y.), 30(6), 063133. https://doi.org/10.1063/5.0004344

Man, K., \& Damasio, A. (2019). Homeostasis and soft robotics in the design of feeling machines. Nature Machine Intelligence, 1(10), 446-452. https://doi.org/10.1038/s42256-019-0103-7

Marr, D. (1983). Vision: A Computational Investigation into the Human Representation and Processing of Visual Information. Henry Holt and Company.

McEliece, R. J., MacKay, D. J. C., \& Jung-Fu Cheng. (1998). Turbo decoding as an instance of Pearl's "belief propagation" algorithm. IEEE Journal on Selected Areas in Communications, 16(2), 140-152. https://doi.org/10.1109/49.661103

Northoff, G. (2012). Immanuel Kant's mind and the brain's resting state. Trends in Cognitive Sciences, 16(7), 356-359. https://doi.org/10.1016/j.tics.2012.06.001

Ocaña, F. M., Suryanarayana, S. M., Saitoh, K., Kardamakis, A. A., Capantini, L., Robertson, B., \& Grillner, S. (2015). The Lamprey Pallium Provides a Blueprint of the Mammalian Motor Projections from Cortex. Current Biology, 25(4), 413-423. https://doi.org/10.1016/j.cub.2014.12.013

Paperin, G., Green, D. G., \& Sadedin, S. (2011). Dual-phase evolution in complex adaptive systems. Journal of the Royal Society Interface, 8(58), 609-629. https://doi.org/10.1098/rsif.2010.0719

Pfeifer, R., \& Bongard, J. (2006). How the Body Shapes the Way We Think: A New View of Intelligence. A Bradford Book.

Ramstead, M. J. D., Kirchhoff, M. D., \& Friston, K. J. (2019). A tale of two densities: Active inference is enactive inference [Preprint]. http://philsci-archive.pitt.edu/16167/

Rudrauf, D., Bennequin, D., Granic, I., Landini, G., Friston, K. J., \& Williford, K. (2017). A mathematical model of embodied consciousness. Journal of Theoretical Biology, 428, 106-131. https://doi.org/10.1016/j.jtbi.2017.05.032

Safron, A. (2019). Bayesian Analogical Cybernetics. ArXiv:1911.02362 [q-Bio]. http://arxiv.org/abs/1911.02362

Safron, A. (2020a). An Integrated World Modeling Theory (IWMT) of Consciousness: Combining Integrated Information and Global Neuronal Workspace Theories With the Free Energy Principle and Active Inference Framework; Toward Solving the 
Hard Problem and Characterizing Agentic Causation. Frontiers in Artificial Intelligence, 3. https://doi.org/10.3389/frai.2020.00030

Safron, A. (2020b). Integrated World Modeling Theory (IWMT) Implemented: Towards Reverse Engineering Consciousness with the Free Energy Principle and Active Inference. PsyArXiv. https://doi.org/10.31234/osf.io/paz5j

Safron, A. (2020c). On the Varieties of Conscious Experiences: Altered Beliefs Under Psychedelics (ALBUS). PsyArXiv. https://doi.org/10.31234/osf.io/zqh4b

Safron, A. (2021a). The Radically Embodied Conscious Cybernetic Bayesian Brain: From Free Energy to Free Will and Back Again. Entropy, 23(6), 783. https://doi.org/10.3390/e23060783

Safron, A. (2021b). Integrated World Modeling Theory (IWMT) Expanded: Implications for Theories of Consciousness and Artificial Intelligence. PsyArXiv. https://doi.org/10.31234/osf.io/rm5b2

Sandrone, S., \& Safron, A. (2013). Pain and (e) motion in postural responses. Frontiers in Human Neuroscience, 7, 286.

Schmidhuber, J. (2020, December). 1990: Planning \& Reinforcement Learning with Recurrent World Models and Artificial Curiosity. AI Blog: Jürgen Schmidhuber. https://people.idsia.ch// juergen/world-models-planning-curiosity-fki-1990.html

Schmidhuber, J. (2021, January). 1991: First very deep learning with unsupervised pre-training. AI Blog: Jürgen Schmidhuber. https://people.idsia.ch// juergen/very-deep-learning-1991.html

Seth, A. K., \& Tsakiris, M. (2018). Being a Beast Machine: The Somatic Basis of Selfhood. Trends in Cognitive Sciences, 22(11), 969-981. https://doi.org/10.1016/j.tics.2018.08.008

Shanahan, M. (2010). Embodiment and the Inner Life: Cognition and Consciousness in the Space of Possible Minds. Oxford University Press.

Shea, N., \& Frith, C. D. (2019). The Global Workspace Needs Metacognition. Trends in Cognitive Sciences, 0(0). https://doi.org/10.1016/j.tics.2019.04.007

Shine, J. M. (2019). Neuromodulatory Influences on Integration and Segregation in the Brain. Trends in Cognitive Sciences, 23(7), $572-$ 583. https://doi.org/10.1016/j.tics.2019.04.002

Shine, J. M., Breakspear, M., Bell, P. T., Martens, K. A. E., Shine, R., Koyejo, O., Sporns, O., \& Poldrack, R. A. (2019). Human cognition involves the dynamic integration of neural activity and neuromodulatory systems. Nature Neuroscience, 22(2), 289.

Solms, M. (2021). The Hidden Spring: A Journey to the Source of Consciousness. W. W. Norton \& Company.

Strindberg, M., Fransson, P., Cabral, J., \& Ådén, U. (2021). Spatiotemporally flexible subnetworks reveal the quasi-cyclic nature of integration and segregation in the human brain. NeuroImage, 239, 118287. https://doi.org/10.1016/j.neuroimage.2021.118287

Suryanarayana, S. M., Pérez-Fernández, J., Robertson, B., \& Grillner, S. (2020). The evolutionary origin of visual and somatosensory representation in the vertebrate pallium. Nature Ecology \& Evolution, 1-13. https://doi.org/10.1038/s41559-020-1137-2

Suryanarayana, S. M., Robertson, B., Wallén, P., \& Grillner, S. (2017). The Lamprey Pallium Provides a Blueprint of the Mammalian Layered Cortex. Current Biology: CB, 27(21), 3264-3277.e5. https://doi.org/10.1016/j.cub.2017.09.034

Swanson, L. R. (2016). The Predictive Processing Paradigm Has Roots in Kant. Frontiers in Systems Neuroscience, 10, 79. https://doi.org/10.3389/fnsys.2016.00079

Tani, J. (2016). Exploring robotic minds: Actions, symbols, and consciousness as self-organizing dynamic phenomena. Oxford University Press.

Taylor, A. H., Knaebe, B., \& Gray, R. D. (2012). An end to insight? New Caledonian crows can spontaneously solve problems without planning their actions. Proceedings. Biological Sciences, 279(1749), 4977-4981. https://doi.org/10.1098/rspb.2012.1998

Tegmark, M. (2017). Life 3.0: Being Human in the Age of Artificial Intelligence. Knopf Doubleday Publishing Group.

Tishby, N., \& Zaslavsky, N. (2015). Deep Learning and the Information Bottleneck Principle. ArXiv:1503.02406 [Cs]. http://arxiv.org/abs/1503.02406

Tononi, G., Boly, M., Massimini, M., \& Koch, C. (2016). Integrated information theory: From consciousness to its physical substrate. Nature Reviews Neuroscience, 17(7), 450. https://doi.org/10.1038/nrn.2016.44

Tononi, G., \& Koch, C. (2015). Consciousness: Here, there and everywhere? Philosophical Transactions of the Royal Society B: Biological Sciences, 370(1668), 20140167. https://doi.org/10.1098/rstb.2014.0167

VanRullen, R., \& Kanai, R. (2021). Deep learning and the Global Workspace Theory. Trends in Neurosciences. https://doi.org/10.1016/j.tins.2021.04.005

Vasas, V., \& Chittka, L. (2019). Insect-Inspired Sequential Inspection Strategy Enables an Artificial Network of Four Neurons to Estimate Numerosity. IScience, 11, 85-92. https://doi.org/10.1016/j.isci.2018.12.009

Wiese, W. (2020). The science of consciousness does not need another theory, it needs a minimal unifying model. Neuroscience of Consciousness, 2020(niaa013). https://doi.org/10.1093/nc/niaa013

Xie, S., Kaiser, D., \& Cichy, R. M. (2020). Visual Imagery and Perception Share Neural Representations in the Alpha Frequency Band. Current Biology, 30(13), 2621-2627.e5. https://doi.org/10.1016/j.cub.2020.04.074

Zhou, J., Cui, G., Zhang, Z., Yang, C., Liu, Z., Wang, L., Li, C., \& Sun, M. (2019). Graph Neural Networks: A Review of Methods and Applications. ArXiv:1812.08434 [Cs, Stat]. http://arxiv.org/abs/1812.08434 